\title{
Investimentos em infraestrutura de transportes e desigualdades regionais no Brasil: uma análise dos impactos do Programa de Aceleração do Crescimento (PAC)"
}

\author{
Transport infrastructure investment and regional \\ inequalities in Brazil: an analysis of the impacts of the \\ Growth Acceleration Program (PAC)
}

GUILHERME JONAS COSTA DA SILVA

HUMBERTO EDUARDO DE PAULA MARTINS

HENRIQUE DANTAS NEDER**

\begin{abstract}
RESUMO: O trabalho tem por objetivo analisar os efeitos dos investimentos em infraestrutura, em particular, de transportes, realizados no âmbito do Programa de Aceleração do Crescimento (PAC) sobre a dinâmica regional brasileira. As hipóteses investigadas no trabalho são: (i) os investimentos em infraestrutura contribuem para o crescimento econômico dos estados brasileiros; (ii) os investimentos em infraestrutura realizados no âmbito do PAC têm contribuído para aumentar as taxas de crescimento econômico dos estados brasileiros, (iii) bem como para alterar a dispersão das taxas de crescimento econômico entre os estados, em particular, a favor dos estados de menor produto. Para tanto, emprega-se a metodologia de dados em painel e bootstrapping para as estimativas e predições realizadas, respectivamente. Os resultados empíricos confirmaram apenas a primeira hipótese, mas não as demais, indicando que o PAC não tem contribuído significativamente para aumentar a taxa média de crescimento do produto per capita dos estados nem para reduzir as desigualdades regionais no país.
\end{abstract}

\footnotetext{
* Este artigo é baseado no texto final da pesquisa realizada no âmbito do Programa de Fomento à Pesquisa em Desenvolvimento Econômico (PDE) do BNDES, em Cooperação com a ANPEC. Os autores agradecem o apoio do PDE/BNDES/ANPEC (2012) e os comentários dos pareceristas anônimos da Revista de Economia Política. Naturalmente, os erros e omissões remanescentes são de nossa inteira responsabilidade.

* Professor Adjunto do Instituto de Economia e Tutor do Grupo PET Economia da Universidade Federal de Uberlândia. Email: guilhermejonas@yahoo.com.br; Professor Associado do Instituto de Economia da Universidade Federal de Uberlândia. Email: hmartins@ufu.br; Professor Titular do Instituto de Economia da Universidade Federal de Uberlândia. Email: hdneder@gmail.com. Submetido: 29/Janeiro/2015; Aprovado: 14/Outubro/2015.
} 
PALAVRAS- CHAVE: Infraestrutura de Transportes; Desigualdades Regionais; Programa de Aceleração do Crescimento (PAC); Dados em Painel; bootstrapping; Brasil.

ABSTRACT: The article analyses the effects of investments in infrastructure - in particular regarding transport sector carried out under the Growth Acceleration Program (PAC) - on the Brazilian regional dynamics. The hypotheses investigated in the study are: (i) infrastructure investments have contributed to the economic growth of the Brazilian states; (ii) PAC's infrastructure investments have helped to increase the economic growth rates of the Brazilian states, (iii) PAC's infrastructure investments have changed the dispersion of economic growth rates between states, in favor of poorer states. We employ the methodology of panel data for the estimates and bootstrapping for predictions. The empirical results only confirmed the first hypotheses, but not the last two ones, indicating that the PAC has not contributed significantly to amplify the average rate of growth of GDP per capita at state level; and also that PAC has not contributed to reduce regional inequalities.

KEYWORDS: Transports Infrastructure; Regional Development; Growth Acceleration Program (PAC); Panel Data; bootstrapping; Brazil.

JEL Classification: H54; O40; C53.

\section{INTRODUÇÃO}

O debate sobre os determinantes do crescimento econômico tem ocupado uma posição relevante na literatura há muito tempo. A constante busca por mecanismos que atinjam, em última instância, o crescimento econômico, vem resultando em um grande número de estudos e sugestões de políticas públicas, das quais se podem destacar os investimentos em infraestrutura, em especial, em infraestrutura de transportes.

No Brasil, a discussão da importância dessas políticas tem lugar de destaque, considerando-se a deterioração da infraestrutura no passado recente, as tentativas de recuperação e ampliação da infraestrutura consubstanciadas no Programa de Aceleração de Crescimento (PAC), e as dificuldades de retomada do crescimento sustentado. Essa temática adquire ainda mais relevância quando se considera uma histórica característica do desenvolvimento econômico brasileiro: o elevado nível de desigualdades regionais.

Nesse contexto, o objetivo geral do trabalho é avaliar os efeitos dos investimentos realizados em infraestrutura, em particular, de transportes, no âmbito do Programa de Aceleração do Crescimento (PAC) sobre a dinâmica regional brasileira, considerando os investimentos dos governos federal e estaduais.

São analisadas três hipóteses: (i) os investimentos em infraestrutura contribuem para o crescimento econômico de longo prazo dos estados brasileiros; (ii) os investimentos em infraestrutura realizados no âmbito do PAC têm contribuído para aumentar das taxas de crescimento econômico dos estados brasileiros, (iii) bem como para alterar a dispersão das taxas de crescimento econômico entre os 
estados, em particular, com aumento das taxas de crescimento do produto daqueles menos desenvolvidos, contribuindo para a redução das desigualdades regionais.

Para avaliar os impactos dos investimentos em infraestrutura de transportes realizados no âmbito do PAC no crescimento do produto per capita dos estados brasileiros, foram realizadas predições com base nos resultados da análise empírica no período de 1986 a 2009, contrapostos aos resultados específicos para o triênio 2007-2009, em que foram realizados investimentos do PAC 1 e aos investimentos programados para o período de 2011 a 2014, no âmbito do PAC 2.

Os resultados, em conjunto, indicam a importância dos investimentos em infraestrutura de transportes para o crescimento econômico e, portanto, seu potencial para redução das desigualdades regionais, mas, ao mesmo tempo, permitem questionar o aproveitamento desse potencial no período recente, inclusive depois da implantação do PAC e, assim, formular sugestões para alterações no padrão de investimentos em infraestrutura no Brasil.

O trabalho está estruturado da seguinte forma: depois desta introdução, na segunda seção são apresentadas a concepção teórica de Hirschman e a abordagem da heterogeneidade estrutural, bem como uma revisão da literatura empírica sobre o tema, com atenção aos trabalhos que analisaram a dinâmica regional brasileira. Em seguida, na terceira seção, faz-se um breve diagnóstico sobre a atual situação da infraestrutura de transportes brasileira, considerando sua heterogeneidade regional.

A quarta seção examina empiricamente os impactos dos investimentos em infraestrutura de transportes no âmbito do PAC. Para tanto, são apresentados os aspectos metodológicos e a base de dados utilizada, bem como os resultados empíricos e sua análise. Por fim, as considerações finais sumarizam os principais resultados e, a partir deles, formulam sugestões de diretrizes para políticas públicas.

\section{REVISÃO DA LITERATURA TEÓRICA E EMPÍRICA}

\section{A Concepção de Hirschman e a Abordagem da Heterogeneidade Estrutural: Uma Breve Revisão da Literatura Teórica}

A temática da infraestrutura e sua relação com o desenvolvimento econômico é importante e recorrente na literatura econômica. Em particular, o debate tem procurado analisar e dimensionar a relação da infraestrutura logística (em termos quantitativos e qualitativos) com a atividade econômica e seu ritmo de crescimento econômico.

$\mathrm{Na}$ literatura de cunho teórico destaca-se a contribuição de A. Hirschman, sintetizada no livro The Strategy of Economic Development, de 1958, publicado no Brasil com o título Estratégias de Desenvolvimento Econômico (Hirschman, 1961). Em sua perspectiva, a infraestrutura apresenta um caráter permissivo para a atividade econômica, ou seja, seu papel mais relevante para a economia não está no peso que representa em termos de produto, mas no fato de que permite e estimula as atividades produtivas. 
Assim, a infraestrutura contribui para facilitar ou dificultar a realização do potencial de investimento e crescimento de uma economia, dependendo do seu nível (estoque) em comparação com o nível de atividade econômica. Essa ideia é desenvolvida por Hirschman (1961, p. 132) da seguinte maneira. Primeiramente, o autor distingue o Capital Fixo Social (CFS) e Atividades Diretamente Produtivas (ADP): O Capital Fixo Social (CFS), identificado como a infraestrutura econômica, compreende os serviços básicos para o funcionamento das Atividades Diretamente Produtivas (ADP).

Em seguida, o autor destaca a convicção generalizada de que infraestrutura de transportes e energia elétrica e o investimento em CFS constituem condições essenciais para o desenvolvimento econômico, afirmando que essa ideia é amplamente aceita por acadêmicos e policy makers. Entretanto, a questão de "até que ponto o investimento em CFS lidera ou acompanha o investimento em ADP" é importante e complexa (Hirschman, 1961, p. 135).

Hirschman (1961, p. 145-146) define dois tipos de trajetórias de desenvolvimento, sendo que cada uma delas tem uma força de indução específica, ao criar tipos distintos de incentivo:

a) A estratégia de desenvolvimento via capacidade excessiva de CFS é "essencialmente permissiva", haja vista que serve para reforçar motivações já existentes: uma expansão de CFS tende a diminuir o custo do investimento em ADP e pode incentivá-lo e aumentá-lo, “dependendo da reação dos empreendedores";

b) A estratégia de desenvolvimento via escassez de CFS significa uma expansão de ADP que ocupa e congestiona a CFS e, portanto, tende a aumentar o custo da produção de ADP, pressionando decisivamente por mais investimento em CFS: "Uma carência reconhecida como tal, obriga a tentar remediá-la, por parte de suas vítimas ou dos que pretendem lucrar com sua eliminação" (Hirschman, 1961, p. 146).

Nessa perspectiva teórica, certo nível mínimo de CFS é pré-requisito para qualquer nível de ADP, mas a partir desse mínimo não é mais necessário que a expansão de CFS preceda a expansão de ADP. Ainda que em regiões ou países desprovidos de CFS o desenvolvimento tenha que se iniciar necessariamente via capacidade excessiva, essa via não é a mais favorável para regiões atrasadas de países menos desenvolvidos que já tenham esse patamar mínimo de CFS.

Nesses casos, uma capacidade excessiva de CFS não obterá bons resultados em termos de incentivos ao investimento em ADP, dadas as dificuldades de sua efetivação. Assim, nessas regiões, é preferível fomentar ou proteger as ADP's antes de expandir o CFS. No caso das regiões dinâmicas desses países, entretanto, o provimento de CFS pode trazer respostas altamente eficientes para o aumento do investimento em ADP.

Após compreender as relações entre investimento público e crescimento do produto, bem como entre o "capital fixo social" e "atividades diretamente produtivas", Hirschman se volta para a chamada "transmissão inter-regional e interna- 
cional do crescimento econômico", contrapondo-se à perspectiva tradicional do "desenvolvimento equilibrado": "O ponto de partida é a constatação de que o crescimento econômico não acontece simultaneamente em toda parte, mas concentra-se em pontos específicos no espaço geográfico, de modo que pode ser considerado não equilibrado" (Hirschman, 1961).

Segundo o autor, os polos de crescimento favorecem o desenvolvimento desigual e apontam para uma configuração de regiões progressistas e regiões atrasadas. Os polos criam dois tipos de efeitos nas regiões sob a sua influência, quais sejam: os efeitos de fluência (favoráveis) e os efeitos de polarização (desfavoráveis).

Os efeitos de fluência mais evidentes são as compras e os investimentos que os polos realizam na região do entorno, o que pode significar um vigoroso impulso para as economias circunvizinhas. Os principais efeitos de polarização são os mecanismos de proteção à indústria do polo e a migração de capital e trabalho qualificado para o polo dinâmico. A contraposição dos efeitos de fluência e de polarização configura as tendências relativas à concentração regional das atividades econômicas.

Diante desse quadro, Hirschman discute a distribuição regional do investimento público, considerado um elemento fundamental de sua teoria, que pode atuar no sentido de reduzir as desigualdades características desse processo de crescimento:

"Em outras palavras, se as forças do mercado, expressas através dos efeitos supracitados, resultarem da vitória temporária dos últimos, a política econômica intervencionista entrará em cena para corrigir a situação. Assim, a política econômica terá uma influência decisiva no decorrer de todo o processo [...] A alocação regional dos investimentos públicos é a maneira mais óbvia pela qual a política econômica influencia as taxas de crescimento do produto das diversas regiões de um país. Nesse sentido, podem-se identificar três padrões principais de alocação: dispersão; concentração em áreas de crescimento econômico; e promoção do desenvolvimento em áreas atrasadas" (Hirschman, 1961, p. 285).

Segundo Hirschman, as necessidades de investimento nas áreas de crescimento acelerado são muito grandes no início, mas com o tempo declinam e, além disso, parte do investimento público pode ser financiada pelos proventos obtidos nas inversões anteriores. Com a permanência das fontes de arrecadação, ocorre um saldo positivo de recursos, que estão disponíveis para promoção do desenvolvimento nas regiões mais atrasadas.

Processa-se, assim, a transição para um padrão de investimento público voltado para promover regiões estagnadas. Nesse ponto, o autor faz um alerta: deve-se buscar fortalecer atividade econômica dessas regiões, visto que a infraestrutura possui um caráter permissivo e, por si só, não garante a promoção do desenvolvimento econômico.

A concepção teórica de Hirschman pode ser apreendida, ao mesmo tempo, como uma confirmação da tendência espacialmente concentradora do crescimento 
econômico e de como são implantadas tentativas de diminuí-la, o que é particularmente relevante para a abordagem de países caracterizados por níveis de desigualdades regionais históricos e elevados, como é o caso dos países latino-americanos, em geral, e do Brasil, em particular.

O conceito de heterogeneidade estrutural, desenvolvido no âmbito do estruturalismo latino-americano (Rodríguez, 2009), e presente nas obras de Aníbal Pinto e Celso Furtado, é especialmente interessante para fundamentar e contextualizar esse debate no âmbito latino-americano, bem como para justificar políticas que visem reduzir as desigualdades regionais no Brasil.

Pinto (2000) discute as implicações da heterogeneidade estrutural das economias latino-americanas para a dinâmica de seu desenvolvimento. $\mathrm{O}$ autor identifica a presença de três "camadas" com níveis diferenciados de produtividade na estrutura produtiva nesses países: uma "primitiva", uma "moderna", identificada como polo, e uma "intermediária", com correspondente diferenciação dos segmentos da população, da estrutura produtiva e do espaço econômico.

Essa heterogeneidade gera uma apropriação desigual dos frutos de progresso técnico, circunscrevendo-os, em grande medida, à população e à área do polo moderno, fazendo com o que os impactos da industrialização substitutiva tomem a forma de uma "tríplice concentração: no nível social, no das camadas econômicas e no nível regional" (Pinto, 2000, p. 575). Esse processo resulta em um reforço da heterogeneidade, que enfraquece as regiões periféricas, fazendo com que o polo dependa da suas próprias forças para crescer, o que agrava ainda mais a concentração e diminui estímulos para o próprio crescimento do polo.

Essa trajetória pode ser identificada com um "estilo de desenvolvimento", em geral, desigual, consumista e dependente nos países latino-americanos, e sua reversão não ocorre espontaneamente, necessitando de políticas públicas (Pinto, 1982, 2000). A ideia de heterogeneidade estrutural apresenta um caráter atual nas economias latino-americanas (Rodríguez, 2009) e está presente em vários trabalhos recentes, como Matteo (2013) e Squeff e Nogueira (2013), que focalizam o Brasil.

Ao longo da obra de Celso Furtado, a concentração regional é destacada como uma característica da economia brasileira desde sua formação, que expressa a condição de heterogeneidade característica do subdesenvolvimento. Sua abordagem apresenta pontos de contato com a visão de Aníbal Pinto, pois Furtado assinala que as desigualdades regionais reforçaram-se durante a industrialização substitutiva no Brasil, limitando a constituição de um mercado interno mais forte e homogêneo.

Celso Furtado atuou na formulação e implantação de políticas regionais, quevisavam à redução das desigualdades, à integração nacional e ao desenvolvimento do país, o que é coerente com sua abordagem teórica. Entretanto, conforme assinala o próprio autor, o deslocamento do motor do crescimento, da formação do mercado interno para a integração com a economia internacional, tende a enfraquecer a interdependência entre as distintas regiões e comprometer a implantação de um projeto nacional (Furtado, 1972, 1992, 2007). 


\section{Revisão da Literatura Empírica}

Vários estudos têm sido desenvolvidos com o objetivo de analisar e dimensionar a relação entre infraestrutura e crescimento econômico. No âmbito internacional, o trabalho de Aschauer (1989) é identificado como precursor nesta linha de pesquisa. Nesse trabalho, o autor analisou os Estados Unidos no período 1949-1985 e concluiu que os gastos públicos em infraestrutura foram responsáveis por ganhos de produtividade de investimentos privados e por fomentar o crescimento econômico.

Banister e Berechman (2001), sumarizando uma série de estudos internacionais, apontam que, em geral, a bibliografia mostra que os incrementos no estoque de infraestrutura aumentam a acessibilidade e impactam positivamente a economia, promovendo uma melhora no emprego e na produtividade, especialmente nas regiões em que a infraestrutura é mais escassa. Os autores ressaltam ainda a importância da formulação de políticas públicas como fator crucial para que os efeitos do investimento em infraestrutura sobre o desenvolvimento econômico sejam os maiores possíveis.

Calderón e Servén (2004) analisam a relação entre o estoque de infraestrutura e a taxa de crescimento do PIB utilizando dados em painel de 121 países, para o período de 1960 a 2000. Os resultados mostram que: a) o estoque de infraestrutura tem um efeito positivo e significativo sobre o crescimento econômico de longo prazo; e b) a quantidade e a qualidade da infraestrutura têm um impacto negativo e significativo sobre a concentração de renda. Os autores ressaltam ainda que os resultados são confiáveis quanto a eventuais causalidades reversas e que as experiências mostram ainda que os resultados encontrados são significativos não só estatisticamente, mas também economicamente. Com base nesses resultados, os autores concluem que o desenvolvimento da infraestrutura pode ser uma questão-chave para reduzir a pobreza e aumentar o nível de renda da população.

Gonzalez, Guasch e Serebrisky (2008) mostram que os retornos de investimentos em infraestrutura são geralmente maiores nas etapas iniciais do desenvolvimento econômico, quando o estoque de infraestrutura é pequeno e tende a diminuir em estágios posteriores, à medida que cresce esse estoque.

No Brasil, os trabalhos de Ferreira $(1994,1996)$ são considerados pioneiros neste tipo de análise. $\mathrm{O}$ autor identifica uma relação forte e positiva entre infraestrutura econômica e o produto de longo prazo na economia brasileira, concluindo que os gastos com infraestrutura afetam positivamente a produtividade e o crescimento do produto, encontrando coeficientes bastante elevados. Ferreira (1996) estima a elasticidade entre estoque de infraestrutura, medido a partir de diversas variáveis, e o Produto Interno Bruto, utilizando cointegração, com foco no período 1980-1993. A elasticidade estimada foi positiva e significativa, em torno de 0,70.

Desde então, esse resultado vem sendo confirmado por outros estudos, com metodologias e bases de dados variadas. Os trabalhos de Rigolon e Piccinini (1997), Ferreira e Malliagros (1998) e Silva et al. (2013), por exemplo, mostram que um aumento no investimento em infraestrutura gera um efeito positivo e significativo para o crescimento do PIB do país. Mais recentemente, alguns estudos têm anali- 
sado a relação entre investimento em infraestrutura e crescimento econômico em âmbito subnacional, ou seja, entre regiões e estados brasileiros.

Barros e Raposo (2002) avaliam o papel da disponibilidade de infraestrutura (transporte, comunicação, energia elétrica e saneamento) em relação às desigualdades regionais, discutindo, assim, seu potencial como instrumento de política de desenvolvimento regional. Os resultados mostram que a maior parte dos indicadores está abaixo do que seria esperado, tanto do Nordeste quanto no Norte. Esses resultados levam os autores a concluir que a disponibilidade de infraestrutura tem contribuído para ampliar as disparidades regionais no Brasil.

Campos e Simões (2011) analisam a relação entre infraestrutura de transportes e a dinâmica do desenvolvimento em bases regionais, procurando relacionar as disparidades econômicas regionais brasileiras à infraestrutura de transportes. Os resultados indicam que o estoque inicial de infraestrutura rodoviária tem impacto negativo sobre o crescimento do produto real per capita, mas a variação do estoque dessa infraestrutura apresenta um impacto positivo. Segundo os autores, esses resultados mostram que os investimentos em infraestrutura rodoviária são, de fato, relevantes para o desenvolvimento econômico estadual.

Os trabalhos de Rocha e Giuberti (2007), Fortunato, Silva e Oreiro (2007) e Bertussi e Ellery Junior (2012) focalizam suas análises nos impactos dos investimentos em infraestrutura sobre o crescimento do produto dos estados brasileiros, considerando a possibilidade de haver algumas diferenças regionais nesses efeitos. Nesses trabalhos, busca-se avaliar até que ponto os impactos do investimento em infraestrutura sobre o crescimento econômico são diferenciados regionalmente, ou seja, se existe uma diferença no padrão e na magnitude da resposta (elasticidade) do investimento em infraestrutura entre, de um lado, regiões ou estados mais desenvolvidos (com maior produto per capita) e, de outro, regiões ou estados menos desenvolvidos (com menor produto per capita).

Em geral, os resultados desses trabalhos mostraram que, nos estados das regiões consideradas mais desenvolvidas (Sul e Sudeste), apenas as despesas com infraestrutura de energia elétrica e comunicações apresentaram um impacto positivo e significativo, enquanto nos estados do grupo das regiões consideradas menos desenvolvidas (Norte e Nordeste) observou-se um comportamento inverso, ou seja, os impactos das despesas em energia e comunicações não foram significativos e os impactos das despesas em transporte foram positivos e significativos.

Em suma, a bibliografia sobre a temática, em geral, aponta que:

I. Os investimentos em infraestrutura são importantes para o crescimento econômico tanto em nível nacional quanto em nível subnacional (regional), podendo constituir um instrumento para estimular o crescimento e para reduzir as desigualdades regionais;

II. A resposta do crescimento econômico aos investimentos e à disponibilidade de infraestrutura é diferente entre as regiões e os estados, sendo maior nos menos desenvolvidos. 


\section{DIAGNÓSTICO DA INFRAESTRUTURA DE TRANSPORTES NO BRASIL E SUA HETEROGENEIDADE REGIONAL}

A preocupação com a infraestrutura de transportes está presente nas políticas públicas desde a época colonial até o período contemporâneo, ainda que a ênfase e a direção tenham variado bastante ao longo do tempo. Os objetivos fundamentais dessas políticas estavam relacionados com o crescimento econômico e a integração do território nacional, mas o desenvolvimento de uma rede inter-regional de transportes no Brasil foi muito lenta e limitada.

No período de 1870 a 1930, os planos apresentados eram essencialmente ferroviários, mas inicia-se uma fase de debate e de "transição", que foi até o início dos anos 1950. O Plano Nacional de Viação, de 1951, consagra o reconhecimento oficial das rodovias como modalidade prioritária de transporte no Brasil, cuja evolução ocorreu em ritmo extraordinariamente rápido, conforme demonstram os dados (Galvão, 1996, p. 195-196).

As explicações para o atraso no desenvolvimento de uma rede inter-regional de transportes no Brasil são diversas. Para Galvão (1996), as verdadeiras razões desse atraso histórico e também para a quase exclusividade da modalidade rodoviária estão ligadas às pequenas dimensões da economia, em especial, do mercado interno brasileiro:

$\mathrm{Na}$ verdade, tanto o melhoramento quanto a expansão física das ferrovias (e, de igual modo, do transporte hidroviário) deixaram de ocorrer simplesmente porque não havia qualquer justificativa econômica para sua melhoria e expansão, dadas as condições sociais, políticas e econômicas do país ainda vigentes quando do surgimento da era rodoviária [...] Com efeito, um nível baixo de renda, uma excessiva concentração dessa renda e da riqueza nacional, bem como um reduzido mercado interno resultam em pequena densidade de tráfego por unidade de área, fazendo com que o transporte rodoviário seja praticamente o único viável dentre as várias modalidades de transporte (Galvão, 1996, p. 203-204).

A situação da infraestrutura logística da economia brasileira não mudou significativamente com o tempo, continua defasada e concentrada, resultado da falta de um planejamento adequado e da incapacidade histórica do governo em financiar seus investimentos(Lacerda, 2005). Os volumes direcionados para o setor reduziram-se significativamente, de 1,8\% para algo em torno de $0,3 \%$ do PIB no período 1975-2009, dos quais metade corresponde à participação dos investimentos em rodovias, de modo que apenas $0,15 \%$ do PIB são aplicados nos demais modais (CNT, 2010).

A explicação para as sucessivas quedas na taxa de investimento (total e em infraestrutura) está ligada ao seguinte quadro: na década de 1980, em meio à crise econômica, alto grau de endividamento externo, aceleração inflacionária, a política econômica volta-se para o curto prazo e para a estabilização de preços. A pro- 
funda crise fiscal erode a capacidade de investimento do Estado, e os investimentos em infraestrutura caem a níveis baixíssimos. Com a Constituição de 1988, acrescenta-se a esse quadro a ausência de vinculação dos recursos tributários a fundos setoriais e a descentralização tributária que transferiu para estados e municípios tributos incidentes sobre combustíveis e sobre a propriedade de veículos automotores (Araújo Júnior, 2006, p. 33).

A década de 1990 é marcada pelo gradual afastamento do Estado de diversos setores da infraestrutura econômica e pela realização de processos de privatização, colocados como alternativa diante da crise do Estado e na esteira da perspectiva neoliberal. Nesse contexto, há a constituição de agências de regulação para vários campos da infraestrutura, dentre elas as de transporte (ANTT e ANTAQ), e o período é marcado pelas baixas taxas de investimento em infraestrutura de transportes.

Conforme a CNT (2010), a situação é relativamente mais crítica nas regiões menos desenvolvidas, cuja qualidade da infraestrutura de transportes é inferior, o que ajuda a explicar o baixo dinamismo econômico e a baixa atratividade destas regiões.

Recentemente, ciente da necessidade de um planejamento para enfrentar e corrigir as distorções regionais no setor de infraestrutura, o governo federal lançou em 2006 o Programa Nacional de Logística e Transportes (PNLT, 2007), que visa orientar os investimentos em infraestrutura de transportes até 2023, a fim de integrar a matriz de transporte brasileira, atualmente concentrada no modal rodoviário, com outros modais (Campos e Simões, 2011). Nesse sentido, o PNLT busca organizar o sistema de transportes brasileiros a partir de vetores logísticos: Amazônico, Centro-Norte, Nordeste Setentrional, Nordeste Meridional, Leste, Centro-Sudeste e Sul, além de cinco vetores internacionais: Arco Norte, Amazonas, Pacífico Norte, Bolívia e Prata-Chile (PNLT, 2007).

Evidentemente, o PNLT está vinculado ao Programa de Aceleração do Crescimento (PAC), em sua primeira (2007-2010) e segunda versão (2011-2014). No PAC I foram previstos investimentos de 503,9 bilhões de reais, sendo mais de 55 bilhões (cerca de $11 \%$ ) destinados aos transportes, distribuídos em diferentes projetos e modais (Macedo, 2011; Bertussi e Ellery Júnior, 2012; Dávila-Fernández, 2015). O PAC II programou investimentos de R $\$ 955$ bilhões no período entre 2011 e 2014, dos quais foram previstos aproximadamente $\mathrm{R} \$ 100$ bilhões para a infraestrutura de transportes (BRASIL, 2010).

Os investimentos realizados no âmbito do PAC 1 contribuíram para a elevação da média anual do investimento total como porcentagem do PIB, que passou de $16 \%$ no período $2004-2006$ para $18 \%$ do PIB no período 2007-2009, segundo dados do IBGE. O segundo período foi marcado por forte crise econômica internacional, com redução das taxas de crescimento na maior parte dos países: segundo dados do Banco Mundial, entre 2004 e 2006 o crescimento acumulado do PIB mundial foi de $11,8 \%$ e o Brasil cresceu 11,9\%, ao passo que, entre 2007 e 2009 , a taxa acumulada de crescimento do PIB mundial foi de 3,3\%, enquanto no Brasil essa taxa foi de $11,0 \%$. Nesse contexto, o papel do PAC pode ter sido relevante, no sentido de sustentar as taxas de crescimento durante a crise mundial. 
Contudo, alguns trabalhos recentes têm identificado certo grau de concentração regional nos investimentos do PAC e, assim, questionado sua importância para a redução das desigualdades regionais no Brasil (Leitão, 2009; Cardoso Jr. et al., 2009; Domingues et al., 2009; Haddad et al., 2011; Macedo, 2011; Bertussi e Ellery Júnior, 2012).

\section{METODOLOGIA, BASE DE DADOS E RESULTADOS EMPÍRICOS}

\section{Metodologia}

Inicialmente, serão estimados modelos estáticos de dados em painel: modelos de regressão linear pooled (empilhado), modelos de efeitos fixos e modelos de efeitos aleatórios. $\mathrm{Na}$ sequência, são apresentadas as estimativas referentes aos modelos de Arellano-Bond (GMM-Difference) e Arellano-Bover/Blundell-Bond (GMM-System) para o conjunto das unidades da federação do país. ${ }^{1}$

Por fim, emprega-se a metodologia de bootstrapping, que é uma técnica de reamostragem utilizada geralmente quando precisamos estimar a variância de um estimador e quando não é possível utilizar métodos estatísticos convencionais. Para tanto, a partir da amostra observada realizam-se diversas replicações amostrais com reposição (podendo estas amostras ser ou não do mesmo tamanho da amostra original) e a partir deste conjunto de amostras selecionadas obterem a distribuição amostral do estimador.

$\mathrm{Na}$ abordagem clássica da teoria da estimação supõe-se uma população com determinada distribuição e com um valor do parâmetro. No bootstrapping a suposição é de que a amostra que se observa é uma boa representação desta população e pode-se "imitar" a construção da distribuição amostral do estimador (realizada convencionalmente a partir de um suposto conhecimento da distribuição na população) a partir de operação de reamostragem com os dados desta amostra.

\section{Base de Dados}

Na Tabela 1 são apresentadas as estatísticas descritivas das variáveis que mensuram os gastos estaduais por função. Os dados mostram que os gastos estaduais destinados para o setor de infraestrutura de transportes representam os maiores valores, bem como as maiores médias. Contudo, os dados apresentam uma grande dispersão dos valores observados, sinalizando em alguma medida as desigualdades regionais desses gastos do Brasil.

\footnotetext{
${ }^{1}$ Mais detalhes sobre a metodologia, consultar Wooldridge (2002).
} 
Tabela 1: Estatísticas Descritivas das Variáveis Utilizadas nos Modelos Econométricos: 1986-2009

\begin{tabular}{lccccc}
\hline \multicolumn{1}{c}{ Variáveis } & $\mathrm{N}$ & Mínimo & Máximo & Média & Coef. Variação \\
\hline ypercap & 624 & 1.436 & 24.357 & 5.492 & 0.599 \\
ginftransp & 624 & 0 & 457.069 .180 .680 & 5.459 .987 .549 & 4.789 \\
ginfenergcom & 624 & 0 & 8.182 .685 .043 & 63.299 .404 & 5.617 \\
ginfssaneam & 624 & 35.900 .709 & 15.877 .304 .688 & 1.177 .536 .687 & 1.682 \\
ginfeduc & 624 & 23.455 .131 & 26.919 .084 .638 & 2.024 .340 .120 & 1.702 \\
lypercap & 624 & 0.362 & 3.193 & 1.555 & 0.347 \\
logginftransp & 619 & 14.569 & 26.848 & 20.352 & 0.091 \\
logginfenercom & 503 & 7.233 & 22.825 & 16.192 & 0.142 \\
logginfssaneam & 624 & 17.396 & 23.488 & 20.181 & 0.056 \\
logginfeduc & 624 & 16.971 & 24.016 & 20.767 & 0.052 \\
\hline
\end{tabular}

Fonte: Elaboração própria a partir dos dados do Ministério da Fazenda - Secretaria do Tesouro Nacional, disponibilizados pelo IPEADATA.

As variáveis utilizadas nos modelos, referentes ao Produto Interno Bruto e gastos com infraestrutura, foram obtidas do banco de dados do Ministério da Fazenda - Secretaria do Tesouro Nacional, disponibilizados pelo IPEADATA, e são deflacionadas pelo índice geral de preços. Os modelos foram estimados considerando o período 1986-2009 (24 observações anuais) e 26 unidades da federação (para consistência das séries, os dados referentes ao estado do Tocantins foram agregados aos dados do estado de Goiás). Em alguns casos foram estimados modelos logarítmicos, sendo obtidos painéis desbalanceados, já que para algumas variáveis explicativas o valor é nulo para alguns estados e períodos de tempo.

De uma forma geral, os modelos estáticos foram estimados com aproximadamente 500 observações, cuja variável dependente é o logaritmo natural do Produto Interno Bruto real per capita ou a taxa de crescimento do produto real per capita das unidades da federação.

As variáveis explicativas dos modelos foram o logaritmo do investimento total e os valores dos logaritmos dos gastos estaduais em infraestrutura de transportes, energia elétrica e telecomunicações, saúde e saneamento e educação. As definições das variáveis são:

- gypcit é o logaritmo da razão das taxas de crescimento do Produto Interno Bruto real per capita em t (yit) e t-1 (yit-1), ou seja, a diferença dos logaritmos das taxas de crescimento do Produto Interno Bruto real per capita em $\mathrm{t}$ (yit) e t-1 (yit-1);

- $\Delta$ gypcit é a aceleração do crescimento do Produto Interno Bruto real per capita em t;

- loginvestit e $\Delta$ loginvestit são respectivamente o logaritmo do volume e da variação do investimento total em t; 
- logginftranspit e $\Delta$ logginftranspit é o logaritmo e a taxa de crescimento do gasto em infraestrutura de transportes (em termos absolutos) para a unidade da federação i e o ano t. Essa variável gasto ou despesa estadual em infraestrutura de transporte será utilizada como proxy do investimento estadual no setor, pois, segundo o IPEA (2012), registra o "valor de todas as operações destinadas à manutenção e funcionamento de serviços públicos, bem como as relacionadas com obras de conservação, adaptação e manutenção de bens móveis e imóveis";

- logginfenergcomit e $\Delta$ logginfenergcomit é o logaritmo e a taxa de crescimento do gasto em infraestrutura de energia e telecomunicações (em termos absolutos) para a unidade da federação i e o ano t;

- logginfssaneamit e $\Delta$ logginfssaneamit é o logaritmo e a taxa de crescimento do gasto em infraestrutura de saúde e saneamento (em termos absolutos) para a unidade da federação i e o ano t;

- logginfeducit e $\Delta$ logginfeducit é o logaritmo e a taxa de crescimento do gasto em infraestrutura de educação (em termos absolutos) para a unidade da federação i e o ano t;

- dtempo refere-se a dummy tempo que testa e controla a quebra estrutural decorrente do processo de estabilização de preços, no qual se assume o valor unitário para o período anterior ao Plano Real e zero para o período pós-Plano Real;

- drinvtransp refere-se à interação entre a dummy de região e a variável logaritmo natural dos gastos em transporte que testa a existência de alguma diferença significativa dos efeitos dos gastos estaduais adicionais em infraestrutura de transportes nas regiões Norte e Nordeste, se comparados aos das demais regiões do país;

- drinvenergcom refere-se à interação entre a dummy de região e a variável logaritmo natural dos gastos em energia e telecomunicações que testa a existência de alguma diferença significativa dos efeitos dos gastos estaduais adicionais em infraestrutura de energia e telecomunicações nas regiões Norte e Nordeste, se comparados aos das demais regiões do país;

- tendência representa o efeito da evolução ou involução do crescimento do PIB per capita compartilhado pelas economias no período em questão.

\section{Resultados Referentes ao Período 1986-2009}

Nas Tabelas $2^{2}$ e $3^{3}$ podem ser observadas as estimações do modelo para as 26

\footnotetext{
${ }^{2}$ As estimações dos modelos de dados em painel estáticos apontam para a escolha do modelo pooled de acordo com acordo com os resultados dos testes Hausman e teste $\mathrm{F}$ (significância dos efeitos fixos individuais).

${ }^{3}$ Neste trabalho, foram estimadas diversas especificações, com o intuito de escolher o modelo que mais se ajustou aos dados, tanto no sentido de significância estatística dos parâmetros como dos resultados
} 
unidades da federação analisadas no período $1986-2009 .{ }^{4}$ Nota-se que a variável de controle denominada investimento total (loginvestit) foi positiva e estatisticamente significativa em todas as estimações, cumprindo seu papel no modelo.

As estimações indicam uma relação positiva e estatisticamente significativa também ao nível de $5 \%$ entre os gastos dos estados com infraestrutura de transportes (logginftransp) e o crescimento do Produto Interno Bruto per capita (ou produto per capita) dos estados brasileiros (gypc). De fato, quanto maiores forem esses gastos, menores serão os custos das empresas, maior a produtividade e, consequentemente, maior o crescimento do produto per capita das economias. Como pode ser observado na Tabela 2, em média, um aumento de $10 \%$ nos gastos dos estados em infraestrutura de transportes é responsável por um aumento de taxa de crescimento do produto per capita de $0,08 \%{ }^{5}$

Nesse sentido, o investimento total e os investimentos estaduais em infraestrutura de transportes apresentam um caráter complementar e estratégico para o desenvolvimento regional do país, ao criar um ambiente adequado para o investimento privado se instalar, produzir de forma competitiva e dinamizar as economias estaduais. Entretanto, os efeitos do investimento total foram bem superiores aos valores observados para os investimentos estaduais, indicando que o investimento total tem um potencial maior para estimular o desenvolvimento regional e o processo de redução da desigualdade regional no Brasil.

Nestes exercícios, foram incluídas diversas variáveis de controle:

- Variável dtempo, que é uma dummy de tempo para controlar a quebra estrutural em 1994, em função do Plano Real;

- Variável de interação, denominada dummy regional (dr), no qual se atribui valor 1 para estados do Norte e Nordeste, com as variáveis de gasto estadual em transporte (drinvtrans) e energia elétrica / telecomunicações (drinvenerg);

- Variável de tendência, que capta o crescimento exógeno devido, principalmente, ao progresso tecnológico e as demais variáveis que apresentam alguma tendência; $\mathrm{e}$

- Ademais, deve-se salientar que todas as variáveis de gasto estadual, exceto o gasto estadual em infraestrutura de transporte, são variáveis de controle nos modelos.

dos testes para os estimadores Arellano-Bond e Arellano-Bover/Blundel-Bond. Os testes realizados foram: i) os de restrição de sobreidentificação de Sargan e Hansen para a verificação da validade do conjunto de instrumentos; e ii) os testes de Arellano-Bond para autocorrelação dos termos de erro da equação em nível e para a adequação geral do modelo.

${ }^{4}$ Os dados do estado do Tocantins foram agregados aos dados para o estado de Goiás para evitar que o desmembramento do estado afetasse a continuidade das séries.

${ }^{5}$ Um aumento de $0,8 \%$ sobre uma taxa de crescimento no nível de $0,03(3 \%)$ significa uma variação de $3 \%$ para $3,024 \%$, o que representa um aumento de 0,024 pontos percentuais sobre a taxa de crescimento dos estados em média. 
Tabela 2: Resultados das Estimações dos Modelos de Regressão com Dados em Painel (Modelos Estáticos) - Variável Dependente:

Crescimento do Produto Per Capita (gypc). Unidades da Federação (Brasil) - 1986-2009

\begin{tabular}{|c|c|c|c|}
\hline VARIABLES & Polled & $\mathrm{EF}$ & EA \\
\hline \multirow[t]{2}{*}{ Loginvestit } & $0.074^{* *}$ & $0.059^{*}$ & $0.074 * * *$ \\
\hline & $(0.027)$ & $(0.032)$ & $(0.027)$ \\
\hline \multirow[t]{2}{*}{ logginftransp } & $0.008 * *$ & $0.012 * * *$ & $0.008 * * *$ \\
\hline & (0.003) & (0.004) & $(0.003)$ \\
\hline \multirow[t]{2}{*}{ logginfenergcom } & -0.001 & -0.001 & -0.001 \\
\hline & $(0.003)$ & (0.003) & (0.003) \\
\hline \multirow[t]{2}{*}{ logginfssaneam } & -0.006 & 0.004 & -0.006 \\
\hline & $(0.006)$ & $(0.010)$ & $(0.006)$ \\
\hline \multirow[t]{2}{*}{ Logginfeduc } & -0.003 & 0.005 & -0.003 \\
\hline & $(0.006)$ & $(0.012)$ & $(0.006)$ \\
\hline \multirow[t]{2}{*}{ drinvtrans } & -0.001 & -0.004 & -0.001 \\
\hline & $(0.003)$ & $(0.006)$ & (0.003) \\
\hline \multirow[t]{2}{*}{ drinvenergcom } & 0.002 & 0.001 & 0.002 \\
\hline & $(0.003)$ & (0.004) & $(0.003)$ \\
\hline \multirow[t]{2}{*}{ Dtempo } & $-0.042 * *$ & $-0.050 * * *$ & $-0.042 * * *$ \\
\hline & $(0.015)$ & $(0.016)$ & (0.015) \\
\hline \multirow[t]{2}{*}{ Tendência } & 0.001 & -0.000 & 0.001 \\
\hline & $(0.001)$ & $(0.001)$ & $(0.001)$ \\
\hline \multirow[t]{2}{*}{ Constant } & $-1.945^{* *}$ & $-1.929 * *$ & $-1.945 * * *$ \\
\hline & (0.705) & (0.719) & $(0.705)$ \\
\hline Observações & 477 & 477 & 477 \\
\hline R-squared & 0.082 & 0.080 & \\
\hline R2 & 0.0843 & & \\
\hline R2adj & 0.0639 & & \\
\hline Número de id & & 26 & 26 \\
\hline $\mathrm{R} 2 \mathrm{w}$ & & 0.0796 & 0.0749 \\
\hline $\mathrm{R} 2 \mathrm{~b}$ & & 0.000319 & 0.320 \\
\hline R2o & & 0.0125 & 0.0816 \\
\hline chi2_haus & & & 5.555 \\
\hline p_haus & & & 0.783 \\
\hline $\mathrm{F}$ & & & 0.64 \\
\hline p_F & & & 0.9111 \\
\hline
\end{tabular}

Fonte: Elaboração própria a partir dos dados do Ministério da Fazenda - Secretaria do Tesouro Nacional, disponibilizados pelo IPEADATA.

(1) Erros-padrão robustos entre parênteses.

(2) * significante a $10 \%$; ** significante a $5 \%$; *** significante a $1 \%$. 
Tabela 3: Resultados das Estimações dos Modelos de Regressão com Dados em Painel (Modelos Dinâmicos) - Variável Dependente:

Crescimento do Produto Per Capita (gypc).Unidades da Federação (Brasil) - 1986-2009.

\begin{tabular}{|c|c|c|}
\hline VARIABLES & GMM Dif & GMM System \\
\hline \multirow[t]{2}{*}{ Loginvestit } & $0.211 * * *$ & 0.105 \\
\hline & (0.059) & $(0.068)$ \\
\hline \multirow[t]{2}{*}{ Logginftransp } & $0.056^{*}$ & $0.036^{*}$ \\
\hline & $(0.028)$ & $(0.020)$ \\
\hline \multirow[t]{2}{*}{ Logginfenergcom } & -0.014 & -0.005 \\
\hline & $(0.025)$ & $(0.022)$ \\
\hline \multirow[t]{2}{*}{ Logginfssaneam } & -0.030 & 0.013 \\
\hline & (0.038) & (0.029) \\
\hline \multirow[t]{2}{*}{ Logginfeduc } & 0.090 & -0.022 \\
\hline & $(0.065)$ & $(0.067)$ \\
\hline \multirow[t]{2}{*}{ Drinvtrans } & -0.033 & 0.003 \\
\hline & $(0.052)$ & $(0.023)$ \\
\hline \multirow[t]{2}{*}{ Drinvenerg } & 0.016 & 0.003 \\
\hline & $(0.041)$ & $(0.032)$ \\
\hline \multirow[t]{2}{*}{ Dtempo } & $-0.309 * * *$ & $-0.255^{* *}$ \\
\hline & $(0.076)$ & $(0.096)$ \\
\hline \multirow[t]{2}{*}{ Tendência } & $-0.016 * * *$ & $-0.010^{* *}$ \\
\hline & $(0.004)$ & $(0.004)$ \\
\hline \multirow[t]{2}{*}{ Constant } & & -3.121 \\
\hline & & (0.989) \\
\hline Observations & 451 & 477 \\
\hline Number of id & 26 & 26 \\
\hline F_p & $1.59 e-09$ & 0.00559 \\
\hline J & 16 & 21 \\
\hline $\operatorname{ar} 1 p$ & 0.00208 & 0.00205 \\
\hline $\operatorname{ar} 2 p$ & 0.648 & 0.844 \\
\hline sargannp1 & 0.933 & 0.293 \\
\hline hansenp2 & 0.738 & 0.211 \\
\hline hansenp2 & & 0.717 \\
\hline Difftest & & 0.042 \\
\hline Transformação & ortogonal & ortogonal \\
\hline Tipo de estimação & difference & system \\
\hline Instrumentos & $\begin{array}{l}\mathrm{L}(1 / 4) \text {. (loginvfed logginftransp } \\
\text { logginfenergcom } \\
\text { logginfssaneam) collapsed }\end{array}$ & $\begin{array}{l}\mathrm{L}(1 / 4) \text {.(loginvfed logginftransp } \\
\text { logginfenergcom } \\
\text { logginfssaneam) collapsed }\end{array}$ \\
\hline
\end{tabular}

Fonte: Elaboração própria a partir dos dados do Ministério da Fazenda - Secretaria do Tesouro Nacional, disponibilizados pelo IPEADATA.

(1) Erros-padrão robustos entre parênteses.

(2) * significante a $10 \%$; * significante a $5 \%$; *** significante a $1 \%$. 
As estimações indicaram ainda que a dummy tempo (dtempo) foi negativa e significativa sugerindo que houve um aumento do crescimento do produto per capita das unidades da federação no subperíodo mais recente, qual seja, após a implantação do Plano Real. Além disso, as variáveis de interação (entre a dummy regional e as despesas por função) não apresentaram significância estatística na determinação do crescimento do produto per capita, sugerindo que, atualmente, não há diferenças significativas nos efeitos dos gastos em infraestrutura em transportes sobre a taxa de crescimento dos estados nas regiões Norte e Nordeste, em relação às demais regiões do país.

\section{Predições dos Impactos do PAC I e PAC II sobre o Desenvolvimento Regional no Brasil}

Os resultados das estimações dos modelos anteriores podem ser utilizados para a realização de predições visando avaliar os impactos do PAC sobre as taxas de crescimento do Produto Interno Bruto per capita dos estados. O objetivo destas predições é verificar se o PAC: a) contribuiu para elevar, em termos médios, os valores destas taxas; e b) contribuiu para alterar a dispersão entre as mesmas, em favor dos estados de menor nível de produto.

Para atingir estes objetivos, foram realizados testes estatísticos e predições. Com os parâmetros dos modelos estimados para o período 1986-2009, foram realizadas as seguintes predições:

1. Predição das taxas de crescimento do Produto Interno Bruto real per capita para o período 2007-2009 a partir dos parâmetros estimados do modelo estático;

2. Predição das taxas de crescimento do Produto Interno Bruto real per capita para o período 2007-2009 a partir dos parâmetros estimados do modelo estático e com os gastos de investimento em infraestrutura de transportes nos estados (médias anuais estaduais para o quadriênio 2011-2014);

3. Predição das taxas de crescimento do Produto Interno Bruto real per capita para o período 2007-2009 a partir dos parâmetros estimados do modelo com estimador Arellano-Bond;

4. Predição das taxas de crescimento do Produto Interno Bruto real per capita para o período 2007-2009 a partir dos parâmetros estimados do modelo com estimador Arellano-Bond e com os gastos de investimento em infraestrutura de transportes nos estados (médias anuais estaduais para o quadriênio 2011-2014).

As predições 1 e 3 são realizadas com os mesmos dados das variáveis independentes observadas no período 2007-2009. As predições 2 e 4 são contrafactuais para o período 2007-2009 com investimentos planejados para o período 2011-2014 (PAC II). Dessa forma, os resultados referentes às diferenças das predições entre $2 \mathrm{e}$ 1 e entre 4 e 3 serão estimativas do impacto diferencial a ocorrer devido ao efeito 
do PAC I e PAC II. Caso o resultado seja positivo significará um efeito diferencial positivo a favor do PAC II em relação ao PAC I.

$\mathrm{Na}$ Tabela 4 é realizado um teste de diferenças de médias das taxas de crescimento do Produto Interno Bruto real per capita observado para os períodos 2004-2006 e 2007-2009. A diferença das médias entre o segundo período e o primeiro período é positiva e favorece este último $(0,0069)$. No entanto, este valor não é significativo ( $p$-value para o teste unilateral a direita é igual 0,2267$)$, indicando que aparentemente o PAC I não produziu efeito significativo para elevara média das taxas de crescimento dos estados, em relação ao período imediatamente anterior.

Tabela 4: Teste de Hipótese de Diferenças das Médias das Taxas de Crescimento do Produto Per Capita para os Períodos 2004-2006 e 2007-2009

\begin{tabular}{|c|c|c|c|c|c|c|}
\hline $\begin{array}{c}\text { Período } \\
\text { (Anos) }\end{array}$ & Obs. & Média & $\begin{array}{c}\text { Erro- } \\
\text {-Padrão }\end{array}$ & $\begin{array}{l}\text { Desvio- } \\
\text {-Padrão }\end{array}$ & \multicolumn{2}{|c|}{ [95\% Intervalo de Confiança] } \\
\hline 2007-2009 & 78 & 0.0385 & 0.0071 & 0.0630 & 0.0243 & 0.0527 \\
\hline 2004-2006 & 78 & 0.0316 & 0.0058 & 0.0511 & 0.0200 & 0.0431 \\
\hline Combinado & 156 & 0.0350 & 0.0046 & 0.0573 & 0.0260 & 0.0441 \\
\hline Diff & & 0.0069 & 0.0092 & & -0.0112 & 0.0251 \\
\hline \multicolumn{7}{|c|}{ Satterthwaite's degrees of freedom $=147.736 \mathrm{t}=0.7518$} \\
\hline \multicolumn{7}{|c|}{ Ha: diff $<0 \quad \operatorname{Pr}(T<\mathrm{t})=0.7733$} \\
\hline \multicolumn{7}{|c|}{ Ha: $\operatorname{diff} \neq 0 \quad \operatorname{Pr}(|T|>|t|)=0.4533$} \\
\hline \multicolumn{7}{|c|}{ Ha: $\operatorname{diff}>0 \quad \operatorname{Pr}(T>t)=0.2267$} \\
\hline
\end{tabular}

Fonte: Elaboração própria a partir dos dados do Ministério da Fazenda - Secretaria do Tesouro Nacional, disponibilizados pelo IPEADATA e dos Relatórios Estaduais do PAC (BRASIL, 2012a, 2012b),

Obs.: O teste foi realizado com as taxas de crescimento do produto per capita observadas para os dois períodos.

$\mathrm{Na}$ Tabela 5 são apresentados os resultados da estimação por bootstrapping da diferença entre os coeficientes de variação para os mesmos dois períodos. Os resultados mostram uma diferença não significativa, indicando que o PAC I não teve um impacto significativo sobre os coeficientes de variação das taxas de crescimento dos estados. Na segunda coluna desta tabela tem-se o valor do coeficiente observado, que corresponde à diferença entre os coeficientes de variação para os dois períodos analisados $(-0,0068)$. Na terceira coluna, coloca-se o valor do erro-padrão estimado pela técnica bootstrapping $(0,1787)$.

Este valor foi obtido através de 10000 replicações amostrais com reposição para os valores das taxas de crescimento. Para cada uma destas replicações foi calculado uma diferença entre coeficientes de variação e, posteriormente, obteve-se o valor do erro-padrão a partir de todas as replicações. O valor do erro-padrão comparado com o valor observado resulta em uma estatística $\mathrm{z}$ (quarta coluna) reduzida $(-0,04)$ e um p-value elevado $(0,970)$, o que indica aceitação da hipótese 
nula de não ocorrência de mudança significativa no coeficiente de variação das taxas de crescimento dos estados, considerando-se os dois períodos.

Tabela 5: Teste de Diferença de Coeficientes de Variação por Bootstrapping (diff = Coeficiente de Variação Período 2007-2009; diff = Coeficiente de Variação

Período 2004-2006) com 10000 Replicações

\begin{tabular}{ccccc}
\hline Variável & $\begin{array}{c}\text { Coeficiente } \\
\text { Observado }\end{array}$ & $\begin{array}{c}\text { Erro-padrão } \\
\text { Bootstrap }\end{array}$ & $z$ & P-value \\
\hline Diff & -0.0068 & 0.1787 & -0.04 & 0.970 \\
\hline
\end{tabular}

Fonte: Elaboração própria a partir dos dados do Ministério da Fazenda - Secretaria do Tesouro Nacional, disponibilizados pelo IPEADATA e dos Relatórios Estaduais do PAC (BRASIL, 2012a; 2012b);

Obs. O teste foi realizado com as taxas de crescimento do produto per capita observadas para os dois períodos.

$\mathrm{Na}$ Tabela 6 são apresentadas algumas estatísticas descritivas calculadas a partir de predições realizadas com os modelos de dados em painel. Dessa forma, foram realizados dois conjuntos de predições: i) predições para o período 2007-2009 realizadas com os parâmetros das regressões estimados; ii) predições para o período 2007-2009 realizadas com os valores médios anuais do PAC II acrescentados aos valores dos investimentos anteriores. Neste segundo conjunto de predições, o objetivo foi a determinação de um contrafactual para o período 2007-2009 supondo-se a vigência do PACII para este período.

Para o modelo estático, a média das predições reduz-se levemente do primeiro $(0,0529)$ para o segundo período $(0,0431)$. Já o coeficiente de variação eleva-se um pouco, de 0,1508 para 0,1755. As predições para o modelo com o estimador Arellano-Bond (predição modelo Arellano-Bond com PAC I e predição modelo Arellano-Bond com PAC II) também mostram um decréscimo em suas médias (de -0,0074 para -0,0128), indicando também que a passagem do PAC I para o PAC II não contribuiu para a elevação das taxas de crescimento dos estados. Por este estimador observa-se uma redução do coeficiente de variação.

Tabela 6: Estatísticas Descritivas das Predições das Taxas de Crescimento do Produto Per Capita para o Período 2007-2009

\begin{tabular}{cccccc}
\hline Variável & N & Mínimo & Máximo & Média & CV \\
\hline Observado & 78 & -0.1171 & 0.2034 & 0.0385 & 1.6376 \\
Predição Modelo Estático com PAC I & 58 & 0.0334 & 0.0689 & 0.0529 & 0.1508 \\
Predição Modelo Estático com PAC II & 58 & 0.0242 & 0.0587 & 0.0431 & 0.1755 \\
Predição Modelo AB com PAC I & 58 & -0.2891 & 0.6279 & -0.0074 & -37.4363 \\
Predição Modelo AB com PAC II & 58 & -0.2902 & 0.6157 & -0.0128 & -22.2354 \\
\hline
\end{tabular}

Fonte: Elaboração própria a partir dos dados do Ministério da Fazenda - Secretaria do Tesouro Nacional, disponibilizados pelo IPEADATA e dos Relatórios Estaduais do PAC (BRASIL, 2012a, 2012b).

Obs.: As predições com PAC I foram realizadas com os valores observados das séries para o período 2007-2009.

As predições com PAC II foram obtidas através de um procedimento contrafactual que consistiu em realizar predições para o período 2007-2009 com os valores dos investimentos (médios anuais) do PAC II "injetados" nos modelos para o período. 
Na Tabela 7 são apresentados resultados de testes de diferenças de valores médios para as taxas de crescimento preditas e testes de diferenças de coeficientes de variação para estas taxas. Estes testes foram realizados através de replicações amostrais (amostras de mesmo tamanho do universo selecionadas com reposição) e obtenção não paramétrica dos valores dos erros padrões para os estimadores destas diferenças.

O resultado correspondente aos modelos dinâmicos aponta que existe diferença significativa ( $\mathrm{p}$-value $=0,009$ ) entre a média dos valores preditos pelos dois procedimentos (predição com os parâmetros estimados pelo modelo dinâmico para o período 2007-2009 e predição contrafactual com os mesmos parâmetros estimados pelo modelo dinâmico mas com os valores do PAC II "injetados" na predição. O mesmo não ocorre quando se analisam os resultados dos testes de diferença de médias de taxas de crescimento preditas quando se utiliza o modelo estático ( $\mathrm{p}$-value $=0,134)$.

Tabela 7: Testes de Diferenças das Médias e Coeficientes de Variação das Taxas de Crescimento Preditas do Produto Per Capita para o Período 2007-2009 Através de Bootstrapping

\begin{tabular}{ccccc}
\hline Variável & $\begin{array}{c}\text { Coeficiente } \\
\text { Observado }\end{array}$ & $\begin{array}{c}\text { Erro-padrão } \\
\text { Bootstrap }\end{array}$ & $z$ & P-value \\
\hline med2est - med1est & 0.056 & 0.037 & 1.500 & 0.134 \\
med2din - med1din & -0.005 & 0.002 & -2.630 & 0.009 \\
\hline
\end{tabular}

Fonte: Elaboração própria a partir dos dados do Ministério da Fazenda - Secretaria do Tesouro Nacional, disponibilizados pelo IPEADATA e dos Relatórios Estaduais do PAC (BRASIL, 2012a, 2012b).

Obs.: m2est-med1est = diferença de médias das taxas de crescimento preditas pelo modelo estático (com PAC II - PAC I); med2din-med1 din = diferença de médias das taxas de crescimento preditas pelo modelo dinâmico (com PAC II - PAC I).

Sumariamente, os resultados das predições dos modelos apontam para as seguintes conclusões:

1. Os resultados indicaram que não existem evidências de aumento das taxas médias de crescimento do produto per capita dos estados quando se passa do triênio imediatamente anterior ao de vigência do PAC I (2004-2006) para o triênio inicial do PAC I (2007-2009), ainda que a média anual do investimento total tenha aumentado de $16 \%$ para $18 \%$ do PIB nos períodos em consideração. Como o segundo período foi marcado por forte crise econômica internacional, o PAC pode ter cumprido um papel de sustentar as taxas de crescimento nos estados, evitando uma queda acentuada;

2. As análises demonstraram também que não existem evidências de mudanças significativas na dispersão relativa das taxas de crescimento do produto per capita dos estados quando se comparam estes dois períodos, nem em favor dos estados de menor PIB, nem em favor dos de maior PIB, indicando que o PAC I não contribuiu para a redução das desigualdades regionais; 
3. Os resultados sinalizaram ainda que não existem efeitos diferenciais significativos do PAC II em relação ao PAC I quando se analisam estes dois aspectos abordados (efeitos sobre o nível médio das taxas de crescimento dos estados e o grau de dispersão relativa entre estas taxas de crescimento do produto per capita dos estados).

Conjugando-se estas conclusões, de forma geral, pode-se inferir que o PAC não conduziu a um processo de ampliação das taxas de crescimento do produto per capita dos estados, embora possa estar contribuindo para sustentar essas taxas em um contexto de crise internacional. Além disso, o PAC não está alcançando resultados efetivos em termos da redução das desigualdades regionais do Brasil, considerando a dispersão entre os estados.

Assim, situando esses resultados na visão teórica de Hirschman (1958), pode-se caracterizar o padrão de investimento em infraestrutura de transportes no Brasil no período recente como um padrão ainda caracterizado pela "concentração nas áreas de crescimento econômico", com foco nas áreas que apresentam "crescimento espontâneo".

Levando em conta o volume de atividade econômica, bem como o elevado e persistente nível de concentração regional das atividades produtivas no Brasil, pode-se sustentar que a política de infraestrutura de investimento em transportes já deveria ter assumido outro padrão, ou seja, ser orientada para a "promoção do desenvolvimento nas áreas estagnadas", como chegou a se ensaiar durante a década de 1970 (Diniz, 1993, 2006; Martins et al.., 2011).

Atualmente, pode-se considerar que o padrão de investimento em infraestrutura de transportes no Brasil está em uma zona de transição entre esses dois padrões, já que os investimentos não têm favorecido a redução das desigualdades regionais, mas também não têm contribuído para aumentá-las.

\section{CONSIDERAÇÕES FINAIS}

A análise desenvolvida ao longo do trabalho permitiu caracterizar os efeitos dos investimentos em infraestrutura, em particular, de transportes, para o crescimento econômico nos estados brasileiros no período recente (de 24 anos) e, assim, dimensionar o potencial desses investimentos para a redução das desigualdades regionais. Nesse contexto, foram avaliados os impactos do atual padrão de investimento em infraestrutura para a dinâmica regional brasileira, considerando os investimentos realizados pelo governo federal e pelos governos estaduais no âmbito do PAC.

As hipóteses investigadas no trabalho foram: (i) os investimentos em infraestrutura contribuem para o crescimento econômico dos estados brasileiros; (ii) os investimentos em infraestrutura realizados no âmbito do PAC têm contribuído para aumentar das taxas de crescimento econômico dos estados brasileiros, (iii) bem como para alterar a dispersão das taxas de crescimento econômico entre os estados em favor dos de menor PIB, e, assim, para reduzir as desigualdades regionais. 
A primeira hipótese foi confirmada pelos resultados das estimações dos modelos de regressão com dados em painel (modelos estáticos e dinâmicos). O conjunto dos investimentos foi relevante para o crescimento dos estados, sendo que tanto o investimento total quanto os investimentos efetivados pelos governos estaduais em infraestrutura de transportes foram importantes para o crescimento econômico dos estados, e, assim, apresentaram um caráter complementar e estratégico para o desenvolvimento regional do país. Entretanto, o investimento total, por apresentar efeitos bem superiores, mostra um potencial maior para estimular o desenvolvimento regional e o processo de redução da desigualdade regional no Brasil.

As duas últimas hipóteses não foram confirmadas pelos testes realizados. $\mathrm{O}$ teste de diferenças de médias das taxas de crescimento do Produto Interno Bruto real per capita observado para os períodos 2004-2006 e 2007-2009 demonstrou que, apesar da diferença das médias entre o segundo período e o primeiro período ser positiva em favor do PAC, esta não foi significativa, ou seja, o PAC I não produziu efeito significativo sobre a média das taxas de crescimento dos estados, em relação ao período imediatamente anterior, contribuindo, entretanto, para sustentar as taxas em um contexto de crise internacional.

Além disso, os resultados da estimação por bootstrapping da diferença entre os coeficientes de variação para os mesmos períodos demonstraram que o PAC I não teve um impacto significativo sobre a dispersão relativa das taxas de crescimento dos estados em relação ao período imediatamente anterior, ou seja, não contribuiu significativamente para a redução das desigualdades regionais.

Os resultados indicaram ainda que não existem diferenças significativas do PAC II em relação ao PAC I, notadamente, no que tange aos efeitos do programa sobre o nível médio das taxas de crescimento dos estados e sobre o grau de dispersão relativa dessas taxas.

Com base nesses resultados, que encontram respaldo na perspectiva de Hirschman, pode-se afirmar que, para ingressar efetivamente em um novo padrão e atingir efeitos significativos na redução das desigualdades regionais, a estratégia de crescimento deve ser aperfeiçoada em dois sentidos.

Para tanto, deve-se aumentar o volume de investimento mais que proporcionalmente nas regiões menos desenvolvidas, o que contribuiria para elevar a taxa de crescimento do produto nessas regiões, já que o impacto do investimento em infraestrutura é similar nas regiões mais e menos desenvolvidas. Esse caminho, entretanto, tem o risco de que a capacidade da infraestrutura não seja efetivamente ocupada, gerando mais capacidade do que o desejável.

Para contornar esse risco, deve-se, simultaneamente, estimular as atividades diretamente produtivas nas regiões menos desenvolvidas, para que se ocupe essa capacidade de infraestrutura, com o intuito de ampliar o impacto sobre o crescimento econômico nessas regiões, que ingressariam, assim, em um ciclo virtuoso capaz de reduzir efetivamente as desigualdades regionais. 


\section{REFERÊNCIAS BIBLIOGRÁFICAS}

ARAÚJO JR, I. T.; RAMOS, F. (2006). “Investimento em Infraestrutura e Crescimento Econômico no Brasil”. Revista Economia e Desenvolvimento (Recife), v. 5, $\mathrm{n}^{\circ}$ 2;

ARELLANO, M. ; BOND, S. (1991). "Some tests of specification for panel data: Monte Carlo evidence and an application to employment equations”. Review of Economic Studies ,v. 58, p. 277-297;

ARELLANO, M. ; BOVER, O. (1995). "Another look at the instrumental-variable estimation of error-components models”. .Journal of Econometrics, v. 68, n. 1, p. 29-52;

ASCHAUER, D. (1989). “Is public expenditure productive?” .Journal of Monetary Economics, v. 23, p. 177-200;

BANISTER, D.; BERECHMAN, Y (2001).“Transport investment and the promotion of economic growth”. Journal of Transport Geography, v. 9, Issue 3, September, p. 209-218.;

BARROS, A. R.; RAPOSO, I. (2002). "Dotação de infraestrutura como limitante para redução de disparidades regionais no Brasil”. ANAIS DO ENCONTRO DA ASSOCIAÇÃO BRASILEIRA DE ESTUDOS REGIONAIS, 2, São Paulo (SP).;

BERTUSSI, G.; ELLERY JUNIOR, R. (2012). “Infraestrutura de transporte e crescimento econômico no Brasil”. Journal of Transport Literature, v. 6, n. 4, p. 101-132;

BLUNDELL, R. W. ; BOND, S. R. (1998). "Initial conditions and moment restrictions in dynamic panel data models".Journal of Econometrics, v. 87, p. 115-143;

BRASIL (2012a). Programa de Aceleração do Crescimento (PAC).Relatórios Estaduais. Brasília;

BRASIL. (2012b). Programa de Aceleração do Crescimento 2 (PAC 2). Relatórios Estaduais. Brasília;

CALDERÓN, C.; SERVÉN, L. (2004). “The Effects of Infrastructure Development on Growth and Income Distribution”. World Bank Policy Research Working Paper Series n. 3400;

CAMPOS, S.; SIMÕES, R. (2011). "Infraestrutura de transportes e determinantes do desenvolvimento no Brasil”. ANAIS DO ENCONTRO NACIONAL DE ECONOMIA, 39. Foz do Iguaçu (PR);

CARDOSO JR., J. (coord.) (2009). Brasil em Desenvolvimento: Estado, Planejamento e Políticas Públicas. Brasília: IPEA.

CONFEDERAÇÃO NACIONAL DO TRANSPORTE (2010). Pesquisa CNT de Rodovias 2010: Relatório Gerencial. Brasília: CNT-SEST-SENAT;

DÁVILA-FERNÁNDEZ (2015) "Desindustrialização e o investimento em infraestrutura como instrumento conciliador de uma política industrial base no Brasil”. Revista de Economia Política, v. 35, n. 3, p. 576-600;

DINIZ, C. (1993). "Desenvolvimento poligonal no Brasil: nem desconcentração, nem contínua polarização”. Nova Economia, v. 3, n. 1, p. 35-64;

DINIZ, C. (2006). “A Busca de um Projeto de Nação: O Papel do Território e das Políticas Regional e Urbana". EconomiA, Selecta, v.7, n.4, p.1-18;

DOMINGUES, E.; MAGALHÃES, A; FARIA, W. (2009). “Infraestrutura, crescimento e desigualdade regional: uma projeção dos impactos dos investimentos do PAC em Minas Gerais”. Pesquisa e Planejamento Econômico, v. 39, n. 1, p. 121-158;.

FERREIRA, P. C.; MALLIAGROS, T. (1998). "Impactos Produtivos da Infraestrutura no Brasil: 19501995”. Pesquisa e Planejamento Econômico, v.28, n. 2, p. 315-338.

FERREIRA, P.C. (1994). “Infraestrutura Pública, Produtividade e Crescimento”. FGV-RJ, Texto para Discussão246;

FERREIRA, P.C. (1996). "Investimento em Infraestrutura no Brasil: fatos estilizados e relações de longo prazo”. Pesquisa e Planejamento Econômico. v. 26 , n. 2, p. 231-252;

FORTUNATO, W.; SILVA, G.; OREIRO, J. L. (2007) Gasto público com infraestrutura, acumulação privada de capital e crescimento de longo prazo: uma avaliação teórica e empírica para o Brasil (1985-2003). Prêmio SOF de monografias;

FURTADO, C. (2009). Formação Econômica do Brasil. São Paulo: Companhia das Letras;

FURTADO, C. (1972). Análise do modelo brasileiro. Rio de Janeiro: Civilização Brasileira; 1972.

FURTADO, C. (1992). Brasil: a construção interrompida. Rio de Janeiro: Paz e Terra. 
GONZALEZ, J.; GUASCH, J. L.; SEREBRISKY, T. (2008) "Improving Logistics Costs for Transportation and Trade Facilitation". World Bank Policy Research Working Paper n. 4558;

HADDAD, E., PEROBELLI, F., DOMINGUES, E. e AGUIAR, M. (2011). “Assessing the ex ante economic impacts of transportation infrastructure policies in Brazil". Journal of Development Effectiveness, v. 3, n. 1, 44-61;

HIRSCHMAN, A. (1958). The Strategy of Economic Development. New Haven: Yale University Press, 1958;

HIRSCHMAN, A. (1961). A Estratégia de Desenvolvimento Econômico. Rio de Janeiro: Fundo de Cultura, 1961;

INSTITUTO BRASILEIRO DE GEOGRAFIA E ESTATÍSTICA - IBGE. Disponível em <http://www. ibge.gov.br> ( Acesso em 02/03/2014);

IPEADATA - INSTITUTO DE PESQUISA ECONÔMICA APLICADA. Disponível em: <http://www. ipeadata.gov.br>. Acesso em: 30 Julho 2014;

LACERDA, S. M. (2005). Investimentos nos Portos Brasileiros: Oportunidades da Concessão da Infraestrutura Portuária. Rio de Janeiro: BNDES Setorial, n. 22;

LEITÃO, K. (2009).A dimensão territorial do Programa de Aceleração do Crescimento: Um estudo sobre o PAC no estado do Pará e o lugar que ele reserva à Amazônia no desenvolvimento do país. Tese (Doutorado em Arquitetura e Urbanismo) - Faculdade de Arquitetura e Urbanismo da Universidade de São Paulo, São Paulo.

MACEDO, G. (2011). Programa de Aceleração do Crescimento (PAC): Natureza, impactos e dinâmica regional. Dissertação (Mestrado em Economia) - Instituto de Economia da Universidade Federal de Uberlândia, Uberlândia.

MARTINS, H., SOUSA, G., CASTRO, B., ÁVILA, A. (2011).“Concentração Espacial das Atividades Produtivas no Brasil: Uma análise em nível municipal”. ANAIS DO ENCONTRO NACIONAL DE ECONOMIA POLÍTICA, 16.;Uberlândia, MG;

MATTEO, M. (2013).Heterogeneidade regional. Brasília, DF: CEPAL. Escritório no Brasil/IPEA, 2013. (Textos para Discussão CEPAL-IPEA, 56) 30p;

MDIC, Ministério do Desenvolvimento Indústria e Comercio Exterior (2012). Disponível em: <http:// www.mdic.gov.br//sitio/>. Acessado em: 22 de out. 2012.;

PINTO, A. (1982). "Estilos de desenvolvimento e realidade latino-americana". Revista de Economia Política, v. 2/1, n. 5, p. 29-88;

PINTO, A. (2000). "Natureza e implicações da 'heterogeneidade estrutural' da América Latina". In: BIELSCHOWSKY, R. (Org.). Cinquenta anos de pensamento da Cepal. Rio de Janeiro: Record;

PNLT. Plano Nacional de Logística e Transportes(2007). Ministério dos Transportes (MT). Brasília;

RIGOLON, F.; PICCININI, M. (1997) “O Investimento em Infraestrutura e a Retomada do Crescimento Econômico Sustentado". Texto para Discussão BNDES, Rio de Janeiro, n. 63.

ROCHA, F.; GIUBERTI, A. (2007) "Composição do gasto público e crescimento econômico: uma avaliação macroeconômica da qualidade dos gastos dos Estados brasileiros”. Economia Aplicada, v. 11, n. 4 , p. $463-485$.

RODRÍGUEZ, O. (2009) O estruturalismo latino-americano. Rio de Janeiro: Civilização Brasileira.

ROODMAN, D. (2006) "How to Do xtabond2: An Introduction to "Difference" and "System" GMM in Stata”. Center for Global Development Working Paper N. 103, Washington.

SILVA, F., MARTINS, F., ROCHA, C.; ARAÚJO, C. (2013) "Investimentos em transportes terrestres causam crescimento econômico? Um estudo quantitativo". Journal of Transport Literature, v. 7, n. 2, 124-145;

SQUEFF, G.;NOGUEIRA, M. (2013) “A heterogeneidade estrutural no Brasil”. Brasília, DF: CEPAL. Escritório no Brasil/IPEA (Textos para Discussão CEPAL-IPEA, 51).

WOOLDRIDGE, J. M. (2002). Econometric Analysis of Cross Section and Panel Data. Cambridge, MA: MIT Press. 